\title{
Erratum to: Data analysis for the NASA EOS Aura Microwave Limb Sounder instrument
}

\author{
W. Van Snyder • Nathaniel J. Livesey • \\ William G. Read • Zvi Shippony
}

Published online: 9 September 2011

C) Springer-Verlag 2011

\section{Erratum to: Int J Geomath DOI 10.1007/s13137-011-0020-3}

In this article, an error crept into Eqs. (16) and (17). The factor $\alpha(s)$ ought not to appear.

Equations (16) and (17), as they appeared, were

$$
\begin{aligned}
\frac{\partial I\left(s_{r}\right)}{\partial f_{l m}^{k}} & =\int_{s_{0}}^{s_{r}} \alpha(s) \mathscr{T}\left(s, s_{r}\right)(B(s)-I(s)) \frac{\partial \alpha(s)}{\partial f_{l m}^{k}} \mathrm{~d} s \\
\frac{\partial I\left(s_{r}\right)}{\partial f_{l m}^{k}} & =\int_{s_{0}}^{s_{r}} \alpha(s) \mathscr{T}\left(s, s_{r}\right)(B(s)-I(s)) \beta^{k}(s) \mu_{l m}^{k}(s) \mathrm{d} s .
\end{aligned}
$$

The factor $\alpha(s)$ ought not to appear. The equations should be

$$
\begin{aligned}
& \frac{\partial I\left(s_{r}\right)}{\partial f_{l m}^{k}}=\int_{s_{0}}^{s_{r}} \mathscr{T}\left(s, s_{r}\right)(B(s)-I(s)) \frac{\partial \alpha(s)}{\partial f_{l m}^{k}} \mathrm{~d} s \\
& \frac{\partial I\left(s_{r}\right)}{\partial f_{l m}^{k}}=\int_{s_{0}}^{s_{r}} \mathscr{T}\left(s, s_{r}\right)(B(s)-I(s)) \beta^{k}(s) \mu_{l m}^{k}(s) \mathrm{d} s .
\end{aligned}
$$

The online version of the original article can be found under doi:10.1007/s13137-011-0020-3.

W. V. Snyder $(\varangle) \cdot$ N. J. Livesey · W. G. Read · Z. Shippony

Jet Propulsion Laboratory, California Institute of Technology, 4800 Oak Grove Drive,

Mail Stop 183-701, Pasadena, CA 91109-8099, USA

e-mail: van.snyder@jpl.nasa.gov 